\title{
A Rare Retroperitoneal Mass: Leiomyosarcoma of the Inferior Vena Cava
}

\author{
Aram Baram*, Zirak Anwar Tayeb, Delan Ahmed Bakir \\ Department of Thoracic and Cardiovascular Surgery, Faculty of Medical Sciences, School of Medicine, \\ University of Sulaimani, François Mitterrand Street, Sulaymaniyah, 46001, Iraq \\ Email: ${ }^{*}$ aram.baramm@gmail.com, dr.zirak@gmail.com, deelan 83@yahoo.com
}

Received 7 February 2014; revised 7 March 2014; accepted 15 March 2014

Copyright @ 2014 by authors and Scientific Research Publishing Inc.

This work is licensed under the Creative Commons Attribution International License (CC BY). http://creativecommons.org/licenses/by/4.0/

\section{Open Access}

\begin{abstract}
Leiomyosarcoma of the inferior vena cava (IVC) is a rare soft tissue tumor, mesenchymal in origin that arises from smooth muscles of tunica media; it accounts for about $0.5 \%$ of all soft tissue sarcomas, and it is the commonest vascular leiomyosarcoma. The tumor progression is slow, and it is asymptomatic until advanced stage in which involvement of surrounding structures even when the symptoms present are nonspecific. Presentation of Case: A 60 years old lady presented with upper abdominal pain for $\mathbf{3}$ months duration. Past surgical history was significant for Hysterectomy 15 years ago. On examination: soft abdomen, palpable non pulsating right hypochondrial mass. Ultrasound of the abdomen showed tumor of the head of pancreas. CT scan showed large retroperitoneal tumor extending from the head of pancreas to IVC. Trans abdominal CT guided FNAC showed retroperitoneal sarcoma while Immunohistochemistry (IHC) was proved to be Leiomyosarcoma of the IVC. Discussion: Leiomyosarcoma of inferior vena cava (IVC) is a rare soft tissue tumor, mesenchymal in origin that arises from smooth muscles of tunica media; it accounts for about $0.5 \%$ of all soft tissue sarcomas, and it is the commonest vascular leiomyosarcoma. The type of surgical management is a matter of debate and includes resection alone, primary repair/cavoplasty, or replacement with a graft. Reconstruction of the IVC is not always required especially in chronic occlusions. Conclusion: Despite all the advanced modalities, surgery remains the most effective method for treatment of Leiomyosarcoma.
\end{abstract}

\section{Keywords}

Ivc Leiomyosarcoma, Retroperitoneal Tumor, Resection

${ }^{*}$ Corresponding author. 


\section{Background}

Leiomyosarcoma of inferior vena cava (IVC) is a rare soft tissue tumor, mesenchymal in origin that arises from smooth muscles of tunica media; it accounts for about $0.5 \%$ of all soft tissue sarcomas [1], and it is the commonest vascular leiomyosarcoma [2].

Patterns of the tumor growth are extraluminal which is the commonest, intraluminal which is rare, around $1 / 3$ of the cases have both extra and intraluminal tumor growth [3].

According to the site involved IVC leiomyosarcomas are divided into 3 levels, Level 1 proximal to the level of Hepatic veins, Level 2 between the level of renal veins and hepatic veins, Level 3 distal to the entry of renal veins to IVC [4]-[6]

The tumor progression is slow [7], and it is asymptomatic until advanced stage in which involvement of surrounding structures and distal metastasis occur [8], even when the symptoms are present are nonspecific, that is why the disease is usually diagnosed after several years [9]. Imaging studies has a major role in diagnosis, demonstration of origin and extension of such tumors [10].

\section{Case Report}

A 60 years old lady presented with upper abdominal pain of 3 months duration, pain was persistent, dull in nature, aggravated by heavy meals, no weight loss. She has long history of Arterial Hypertension. Past surgical history significant for Hysterectomy 15 years before presentation, for which details are not known, results of the biopsy are not available, cancers are not known in the family.

On examination: soft abdomen, audible bowel sounds, palpable non pulsating right hypochondrial mass, Hepatomegaly. Initial laboratory investigations including CBC and Biochemical tests were within normal limits. Ultrasound of the abdomen showed Tumor of the head of Pancreas. CT scan showed large retroperitoneal tumor extending from the head of pancreas to IVC, both renal veins and caudate lobe of the liver [Figure 1]. Trans abdominal CT guided FNAC showed retroperitoneal sarcoma while by Immunohistochemistry (IHC) proved to be Leiomyosarcoma of the IVC. IVC venogram showed subtotal occlusion of IVC with a floating filling defect in the lumen [Figure 2].

She disappeared from follow-up because she went to another institution where high risk resection decided with cardiopulmonary bypass and repair by graft decided for which a midline laparotomy performed, unfortunately intraoperative decision was to terminate the operation because the mass found was not respectable.

In front of this disappointment she returned back to our center, few days after arrival, she was to be found tired, with tachypnea and jaundice and huge swelling of both lower limbs in Phlegmasiaalbadolens, Doppler study of both lower limbs showed extensive DVT reaching IVC.

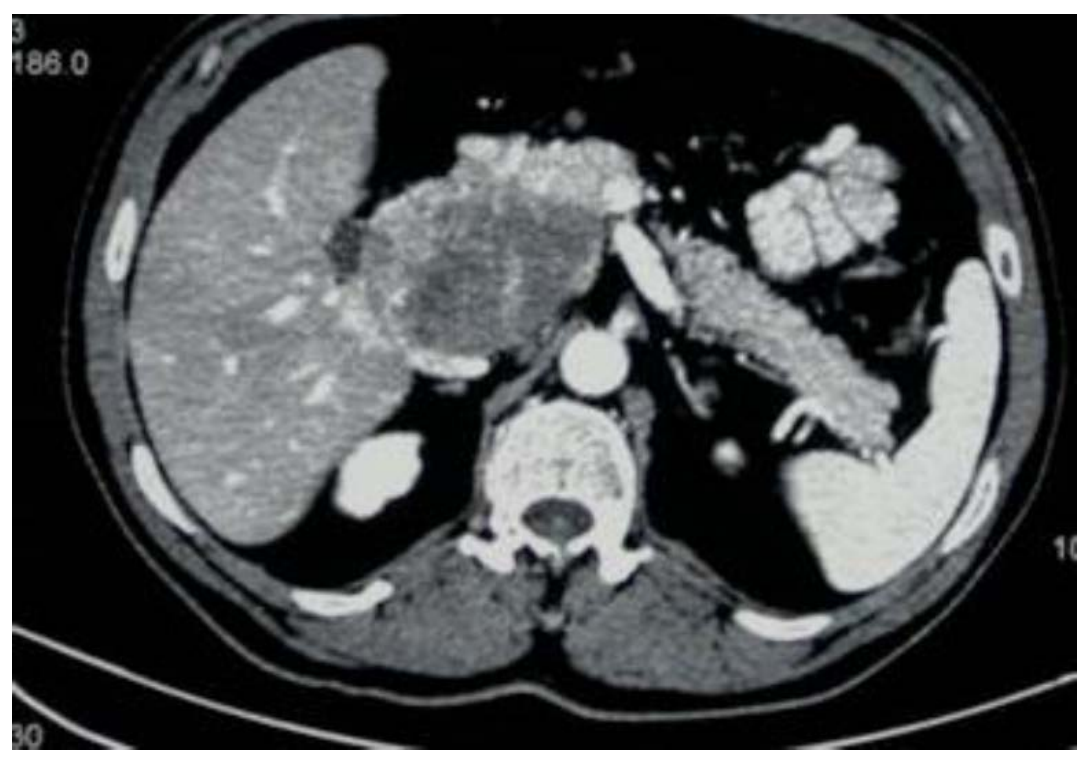

Figure 1. CT-scan of Abdomen showing large retroperitoneal mass. 


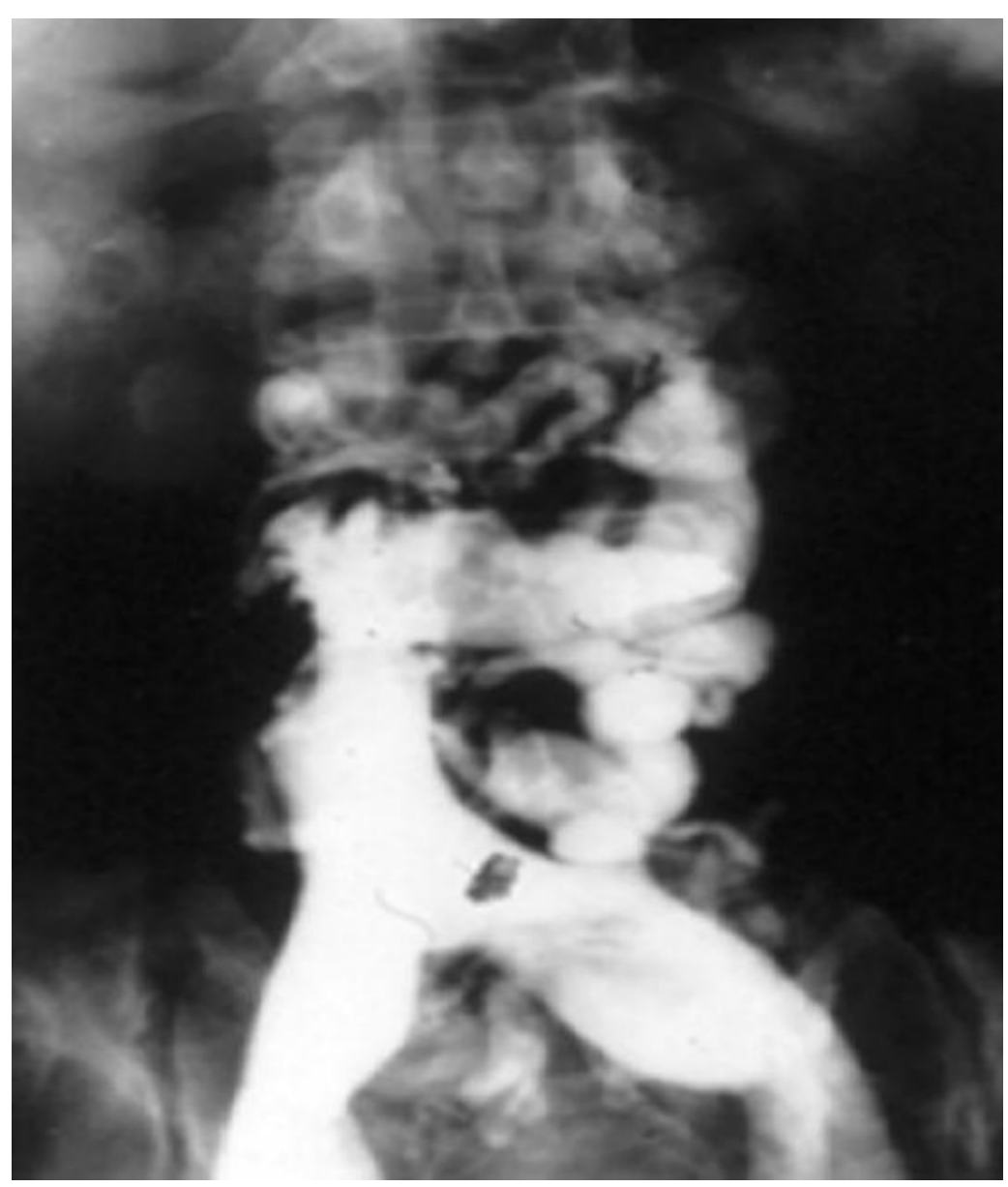

Figure 2. Cavography showing subtotal obstruction with floating endoluminal filling defect.

Oncologist and Vascular Surgeon care started, the patient received 6 cycles of chemotherapy along with systemic anticoagulation and compression stocking of lower limbs. At 11 months later, after down-staging of the tumor, clinical examination revealed no palpable mass, multiple large dilated anterior and posterior abdominal wall veins, and performance status was one. New CT-scan of abdomen showed more than $50 \%$ reduction in the tumor size [Figure 3] and radiological signs of respectability. Doppler study shows chronic DVT, Mild Post Post-thrombotic syndrome, OGD was normal, IVU showed right kidney hydronephrosis. Resection of the residual tumor decided.

\section{Operation}

Through the previous midline laparotomy abdomen opened, tumor noted to originate from IVC, resectability assessed, dissection of the mass from aorta, duodenum, portal vein, caudate lobe of the liver and right kidney done. Proximal and distal control done, Resection of the mass done en-bloc with renal veins and IVC, tumor margins at the time of resection were negative. Postoperative sample examination revealed tumor free excisional margins.

\section{Post-Operative Period}

After the surgery she developed high output renal failure (urine output about $3000 \mathrm{ml} / 24 \mathrm{hrs}$.) with elevation of serum creatinine which reached $3.9 \mathrm{mg} / \mathrm{dl}$. At 5 th post-operative day renal function returned to normal. Systemic anticoagulation was administered through out perioperative period. She discharged from hospital at 9th postoperative day. 


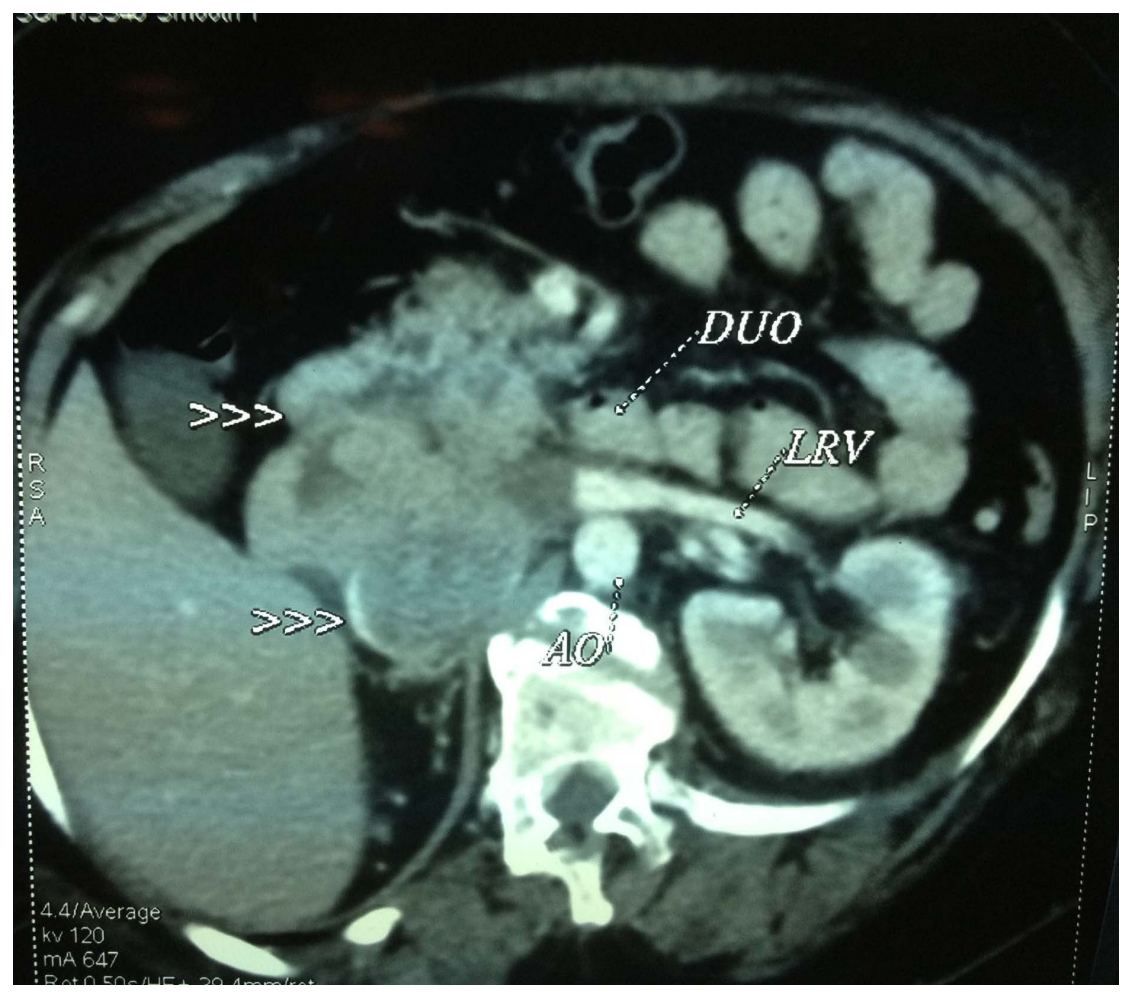

Figure 3. CT-Scan of Abdomen showing regression of tumor size after receiving chemotherapy.

\section{Discussion}

Leiomyosarcoma of inferior vena cava (IVC) is a rare soft tissue tumor, mesenchymal in origin that arises from smooth muscles of tunica media; it accounts for about $0.5 \%$ of all soft tissue sarcomas [1], and it is the commonest vascular leiomyosarcoma [2].

Patterns of the tumor growth are extraluminal which is the commonest, intraluminal which is rare, around 1/3 of the cases have both extra and intraluminal tumor growth [3].

According to the site involved IVC leiomyosarcomas are divided into 3 levels, level 1 proximal to the level of Hepatic veins, level 2 between the level of renal veins and hepatic veins, level 3 distal to the entry of renal veins to IVC [4]-[6].

The tumor progression is slow [7], and it is asymptomatic until advanced stage in which involvement of surrounding structures and distal metastasis occur [8], even when the symptoms present are nonspecific such as dyspnea, malaise, weight loss, abdominal pain, or back pain, that is why the disease is usually diagnosed after several years [9]. Imaging studies have a major role in diagnosis, demonstration of origin and extension of such tumors [10].Computed tomography (CT), magnetic resonance imaging (MRI), individually or in combination with cavography, ultrasound (US), and echocardiography, allow an early and accurate preoperative diagnosis [9].

Despite advancing imaging technology, biopsy is still indicated for formal diagnosis. Histopathology of leiomyosarcoma reveals spindle tumor cells, which are positive for markers of smooth muscle activity includingvimentin, muscle actin, alpha-smooth muscle actin, and desmin [11].

Chemotherapy, using adriamycin-ifosfamide or chemotherapy combined with radiation therapy offers potential adjuvant treatment options [4].

The surgical management of partial resections of the IVC is a matter of current debate and includes ligation, primary repair/cavoplasty, or replacement with a graft. Reconstruction of the IVC is not always required, because gradual occlusion of the IVC allows the development of venous collaterals. However, when pararenal leiomyosarcoma of the IVC is present, reconstruction of the IVC and the renal vein are necessary to prevent transient or permanent renal dysfunction [1]. 
In summary, despite all advanced modalities, surgery remains the most important method for treatment for Leiomyosarcoma.

\section{Conflict of Interest}

No conflict of interest attributed.

\section{Consent}

Written informed consent was obtained from the patient for publication of this case report and any accompanying images. A copy of the written consent is available for review by the editor of this journal.

\section{References}

[1] Alexander, A., Rehders, A., Raffel, A., Poremba, C., Knoefel, W.T. and Eisenberger, C.F. (2009) Leiomyosarcoma of the Inferior Vena Cava: Radical Surgery and Vascular Reconstruction. World Journal of Surgical Oncology, 7, 56. http://dx.doi.org/10.1186/1477-7819-7-56

[2] Ahluwalia, A., Saggar, K., Sandhu, P. and Gupta, K. (2002) Primary Leiomyosarcoma of Inferior Venacava: An Unusual Entity. Indian Journal of Radiology and Imaging, 12, 515-516.

[3] Hartman, D.S., Hayes, W.S., Choyke, P.L. and Tibbets, G.P. (1992) From the Archives of the AFIP. Leiomyosarcoma of the Retroperitoneumand Inferior Vena Cava: Radiologic-Pathologic Correlation. Radio Graphics, 12, 1203-1220. http://dx.doi.org/10.1148/radiographics.12.6.1439022

[4] Kieffer, E., Alaoui, M., Piette, J.C., Cacoub, P. and Chiche, L. (2006) Leiomyosarcoma of the Inferior Vena Cava: Experience in 22 Cases. Annals of Surgery, 244, 289-295. http://dx.doi.org/10.1097/01.sla.0000229964.71743.db

[5] Huguet, C., Ferri, M. and Gavelli, A. (1995) Resection of the Suprarenal Inferior Vena Cava. The Role of Prosthetic Replacement. Archives of Surgery, 130, 793-797. http://dx.doi.org/10.1001/archsurg.1995.01430070115025

[6] Ito, H., Hornick, J.L., Bertagnolli, M.M., George, S., Morgan, J.A., Baldini, E.H., Wagner, A.J., et al. (2007) Leiomyosarcoma of the Inferior Vena Cava: Survival after Aggressive Management. Annals of Surgical Oncology, 14, 3534-3541. http://dx.doi.org/10.1245/s10434-007-9552-z

[7] Bailey, R.V., Stribling, J., Weitzner, S. and Hardy, J.D. (1976) Leiomyosarcoma of the Inferior Vena Cava: Report of a Case and Review of Literature. Annals of Surgery, 184, 169-173.

http://dx.doi.org/10.1097/00000658-197608000-00006

[8] Singh-Panghaal, S., Karcnik, T.J., Wachsberg, R.H. and Baker, S.R. (1997) Inferior Vena Cava Leiomyosarcoma: Diagnosis and Biopsy with Color Doppler Sonography. Journal of Clinical Ultrasound, 25, 275-278. http://dx.doi.org/10.1002/(SICI)1097-0096(199706)25:5<275::AID-JCU10>3.0.CO;2-I

[9] Gowda, R.M., Gowda, M.R., Mehta, N.J., Osborne, R., Bixon, R., Vasavada, B.C. and Sacchi, T.J. (2004) Right Atrial Extension of Primary Venous Leiomyosarcoma: Pulmonary Embolism and Budd-Chiari Syndrome at Presentation-A Case Report. Angiology, 55, 213-216. http://dx.doi.org/10.1177/000331970405500215

[10] Ceyhan, M., Danaci, M., Elmali, M. and Ozmen, Z. (2007) Leiomyosarcoma of the Inferior Vena Cava. Diagn Interv Radiol, 13, 140-143.

[11] Reddy, V.P., et al. (2010) Leiomyosarcoma of the Inferior Vena Cava: A Case Report and Review of the Literature. Cases Journal, 3, 71. 